\title{
Macroscopic evidence for quantum criticality and field-induced quantum fluctuations in cuprate superconductors
}

\author{
A. D. Beyer, ${ }^{1}$ V. S. Zapf, ${ }^{2}$ H. Yang, ${ }^{1}$ F. Fabris, ${ }^{2}$ M. S. Park, ${ }^{3}$ K. H. Kim, ${ }^{3}$ S.-I. Lee, ${ }^{3}$ and N.-C. Yeh ${ }^{1}$ \\ ${ }_{1}^{1}$ Department of Physics, California Institute of Technology, Pasadena, California 91125, USA \\ ${ }^{2}$ National High Magnetic Field Laboratory, Los Alamos, New Mexico 87545, USA \\ ${ }^{3}$ Department of Physics, Pohang University of Science and Technology, Pohang, Korea
}

(Received 15 January 2007; revised manuscript received 7 October 2007; published 25 October 2007)

\begin{abstract}
We present macroscopic experimental evidence for field-induced microscopic quantum fluctuations in different hole- and electron-type cuprate superconductors with varying doping levels and numbers of $\mathrm{CuO}_{2}$ layers per unit cell. The significant suppression of the zero-temperature in-plane magnetic irreversibility field relative to the paramagnetic field in all cuprate superconductors suggests strong quantum fluctuations due to the proximity of the cuprates to quantum criticality.
\end{abstract}

DOI: 10.1103/PhysRevB.76.140506

High-temperature superconducting cuprates are extreme type-II superconductors that exhibit strong thermal, disorder, and quantum fluctuations in their vortex states. ${ }^{1-9}$ While much research has focused on the macroscopic vortex dynamics of cuprate superconductors with phenomenological descriptions, ${ }^{1-5,7}$ little effort has been made to address the microscopic physical origin of their extreme type-II nature. ${ }^{9}$ Given that competing orders (COs) can exist in the ground state of these doped Mott insulators besides superconductivity (SC), ${ }^{9-15}$ the occurrence of quantum criticality may be expected. ${ }^{11,13,16}$ The proximity to quantum criticality and the existence of COs can significantly affect the low-energy excitations of the cuprates due to strong quantum fluctuations ${ }^{8,9}$ and the redistribution of quasiparticle spectral weight among SC and COs. ${ }^{9,17,18}$ Indeed, empirically the low-energy excitations of cuprate superconductors appear to be unconventional, exhibiting intriguing properties unaccounted for by conventional Bogoliubov quasiparticles. ${ }^{9}, 17-19$ Moreover, external variables such as temperature $(T)$ and applied magnetic field $(H)$ can vary the interplay of SC and COs, such as inducing or enhancing ${ }^{20,21}$ the COs at the price of more rapid suppression of SC, thereby leading to weakened superconducting stiffness and strong thermal and field-induced fluctuations. ${ }^{1-3}$ On the other hand, the quasi-two-dimensional nature of the cuprates can also lead to quantum criticality in the limit of decoupling of $\mathrm{CuO}_{2}$ planes. ${ }^{6}$ In this work we demonstrate experimental evidence from macroscopic magnetization measurements for field-induced quantum fluctuations among a wide variety of cuprate superconductors with different microscopic variables such as the doping level $(\delta)$ of holes or electrons, and the number of $\mathrm{CuO}_{2}$ layers per unit cell $(n){ }^{22}$ We suggest that the manifestation of strong fieldinduced quantum fluctuations is consistent with a scenario that all cuprates are in close proximity to a quantum critical point (QCP). ${ }^{6}$

To investigate the effect of quantum fluctuations on the vortex dynamics of cuprate superconductors, our strategy involves studying the vortex phase diagram at $T \rightarrow 0$ to minimize the effect of thermal fluctuations, and applying magnetic field parallel to the $\mathrm{CuO}_{2}$ planes $(H \| a b)$ to minimize the effect of random point disorder. The rationale for having $H \| a b$ is that the intrinsic pinning effect of layered $\mathrm{CuO}_{2}$ planes generally dominates over the pinning effects of ran- dom point disorder, so that the commonly observed glassy vortex phases associated with point disorder for $H \| c$ (e.g., vortex glass and Bragg glass $\left.{ }^{1,5,7}\right)$ can be prevented. In the absence of quantum fluctuations, random point disorder can cooperate with the intrinsic pinning effect to stabilize the low-temperature vortex smectic and vortex solid phases, ${ }^{4}$ so that the vortex phase diagram for $H \| a b$ would resemble that of the vortex-glass and vortex-liquid phases observed for $H \| c$ with a glass transition $H_{G}(T=0)$ approaching $H_{c 2}(T$ $=0$ ). On the other hand, when field-induced quantum fluctuations are dominant, the vortex phase diagram for $H \| a b$ will deviate substantially from predictions solely based on thermal fluctuations and intrinsic pinning, and we expect strong suppression of the magnetic irreversibility field $H_{i r r}^{a b}$ relative to the upper critical field $H_{c 2}^{a b}$ at $T \rightarrow 0$, because the induced persistent current circulating along both the $c$ axis and the $a b$ plane can no longer be sustained if field-induced quantum fluctuations become too strong to maintain the $c$-axis superconducting phase coherence.

In this Rapid Communication we present experimental results that are consistent with the notion that all cuprate superconductors exhibit significant field-induced quantum fluctuations as manifested by a characteristic field $H_{i r r}^{a b}(T \rightarrow 0)$ $\equiv H^{*} \ll H_{c 2}^{a b}(T \rightarrow 0)$. Moreover, we find that we can express the degree of quantum fluctuations for each cuprate in terms of a reduced field $h^{*} \equiv H^{*} / H_{c 2}^{a b}(0)$, with $h^{*} \rightarrow 0$ indicating strong quantum fluctuations and $h^{*} \rightarrow 1$ referring to the mean-field limit. Most important, the $h^{*}$ values of all cuprates appear to follow a trend on an $h^{*}(\alpha)$ vs. $\alpha$ plot, where $\alpha$ is a material parameter for a given cuprate that reflects its doping level, electronic anisotropy, and charge imbalance if the number of $\mathrm{CuO}_{2}$ layers per unit cell $n$ satisfies $n \geqslant 3 .{ }^{23,24}$ In the event that $H_{c 2}^{a b}(0)$ exceeds the paramagnetic field $H_{p}$ $\equiv \Delta_{\mathrm{SC}}(0) /\left(\sqrt{2} \mu_{B}\right)$ for highly anisotropic cuprates, where $\Delta_{\mathrm{SC}}(0)$ denotes the superconducting gap at $T=0, h^{*}$ is defined by $\left(H^{*} / H_{p}\right)$ because $H_{p}$ becomes the maximum critical field for superconductivity.

To find $h^{*}$, we need to determine both the upper critical field $H_{c 2}^{a b}(T)$ and the irreversibility field $H_{i r r}^{a b}(T)$ to as low a temperature as possible. Empirically, $H_{c 2}^{a b}(T)$ can be derived from measuring the magnetic penetration depth in pulsed fields, with $H_{c 2}^{a b}(0)$ extrapolated from $H_{c 2}^{a b}(T)$ values obtained at finite temperatures. The experiments involve measuring 

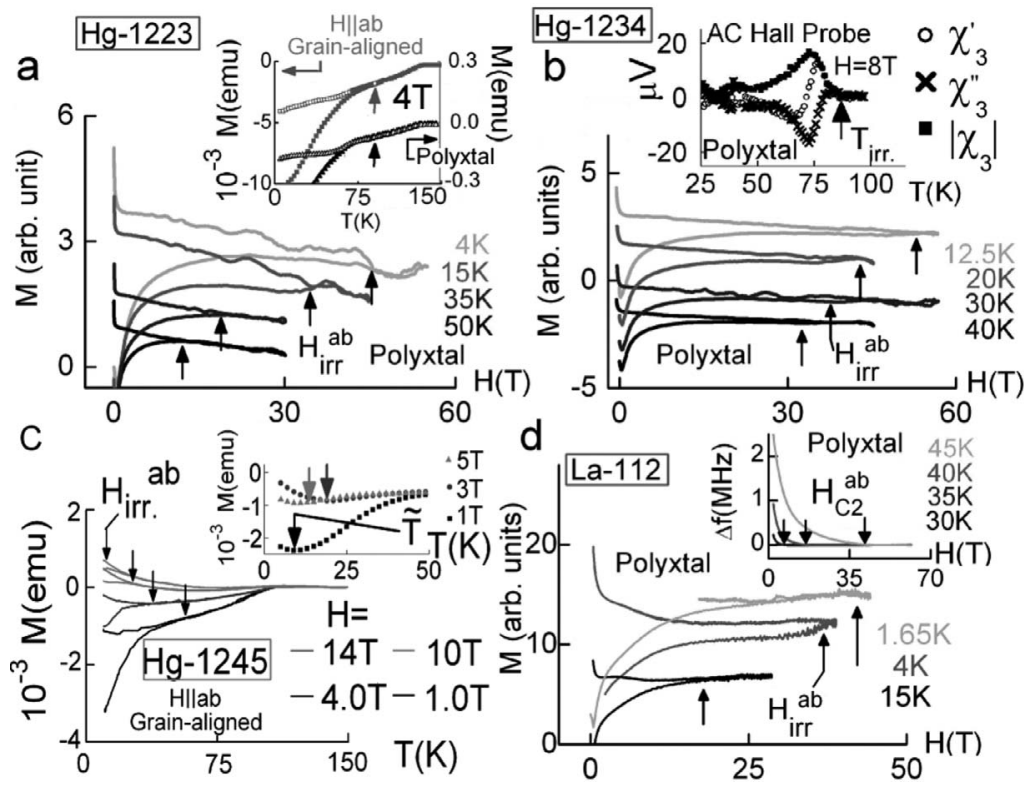

FIG. 1. Representative measurements of the in-plane irreversibility fields $H_{i r r}^{a b}(T)$ in cuprate superconductors: (a) Hg-1223 (polycrystalline and grain aligned), (b) Hg-1234 (polycrystalline), (c) Hg-1245 (grain aligned), and (d) La-112 (polycrystalline and grain aligned). Insets: (a) Consistent $T_{i r r}^{a b}(H)$ obtained from maximum irreversibility of a polycrystalline sample and from irreversibility of a grainaligned sample with $H \|_{a b}$; (b) representative $\chi_{3}$ data taken using ac Hall probe techniques (Ref. 26); (c) details of the $M$ vs $T$ curves, showing an anomalous upturn at $T<\tilde{T}$; (d) exemplifying determination of $H_{c 2}^{a b}$ in La-112 using TDO to measure $\Delta f$ (Ref. 8). the frequency shift $\Delta f$ of a tunnel diode oscillator (TDO) resonant tank circuit with the sample contained in one of the component inductors. Details of the measurement techniques have been given in Ref. 8. In general, we find that the condition $H_{c 2}^{a b}(0)>H_{p}$ is satisfied among all samples investigated so that we define $h^{*} \equiv\left(H^{*} / H_{p}\right)$ hereafter. On the other hand, determination of $H_{c 2}^{a b}(0)$ and $H_{c 2}^{c}(0)$ is still useful because it provides the electronic anisotropy $\gamma \equiv\left(\xi_{a b} / \xi_{c}\right)$ $=\left[H_{c 2}^{a b}(0) / H_{c 2}^{c}(0)\right]$, where $\xi_{a b}\left(\xi_{c}\right)$ refers to the in-plane (c-axis) superconducting coherence length.

To determine $H_{i r r}^{a b}(T)$, we employed different experimental techniques, including dc measurements of the magnetization $M(T, H)$ with the use of a superconducting quantum interference device SQUID magnetometer or a homemade Hall probe magnetometer for lower fields (up to $9 \mathrm{~T}$ ), a dc magnetometer (up to $14 \mathrm{~T}$ ) at the National High Magnetic Field Laboratory (NHMFL) in Los Alamos (LANL-PPMS), a cantilever magnetometer at the NHMFL in Tallahassee for higher fields (up to $33 \mathrm{~T}$ dc fields in a ${ }^{3} \mathrm{He}$ refrigerator), ${ }^{25}$ and a compensated coil for magnetization measurements in the pulsed-field facilities at LANL for even higher fields (up to $65 \mathrm{~T}$ pulsed fields in a ${ }^{3} \mathrm{He}$ refrigerator). ${ }^{8}$ In addition, ac measurements of the third-harmonic magnetic susceptibility $\left(\chi_{3}\right)$ as a function of temperature in a constant field are employed to determine the onset of nonlinearity in the lowexcitation limit. ${ }^{26}$ Examples of the measurements of $H_{i r r}^{a b}(T)$ for $\mathrm{HgBa}_{2} \mathrm{Ca}_{2} \mathrm{Cu}_{3} \mathrm{O}_{\mathrm{x}} \quad\left(\mathrm{Hg}-1223, \quad T_{c}=133 \mathrm{~K}\right)$, $\mathrm{HgBa}_{2} \mathrm{Ca}_{3} \mathrm{Cu}_{4} \mathrm{O}_{\mathrm{x}}\left(\mathrm{Hg}-1234, T_{c}=125 \mathrm{~K}\right), \mathrm{HgBa}_{2} \mathrm{Ca}_{4} \mathrm{Cu}_{5} \mathrm{O}_{\mathrm{x}}$ $\left(\mathrm{Hg}-1245, \quad T_{c}=108 \mathrm{~K}\right)$, and $\mathrm{Sr}_{0.9} \mathrm{La}_{0.1} \mathrm{CuO}_{2}\left(\mathrm{La}-112, T_{c}\right.$ $=43 \mathrm{~K})$, are shown in Figs. 1(a)-1(d), and the consistency among $H_{i r r}^{a b}(T)$ deduced from different techniques have been verified, as summarized in the $H$ vs $T$ phase diagrams $(H \| a b)$ in Figs. 2(a)-2(d). The Hg-based cuprates are in either polycrystalline or grain-aligned forms, and the quality of these samples is confirmed with $\mathrm{x}$-ray diffraction and magnetization measurements to ensure single phase and nearly $100 \%$ volume superconductivity. ${ }^{27,28}$ We have also verified that $H_{i r r}^{a b}(T)$ obtained from the polycrystalline samples are consistent with those derived from the grain-aligned samples with $H \| a b$, because the measured irreversibility in a polycrystalline sample is manifested by its maximum irreversibil-

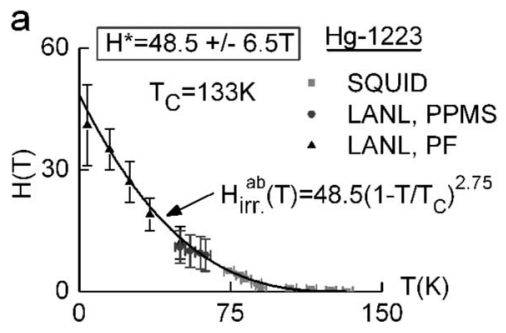

c

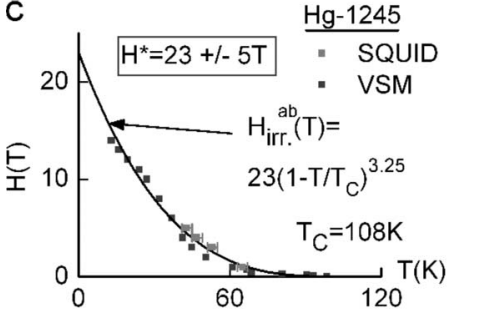

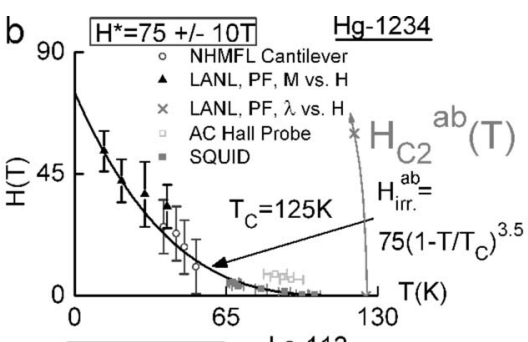

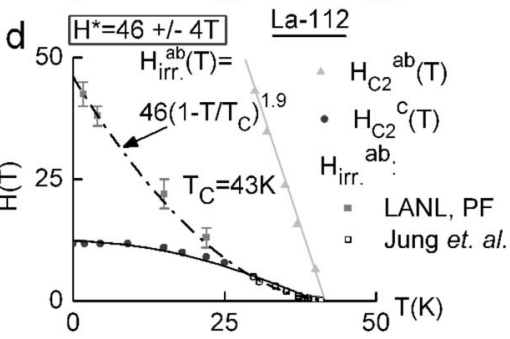

FIG. 2. Determination of $H^{*}$ using various magnetic measurements of $H_{i r r}^{a b}(T)$ : (a) Hg-1223, (b) Hg-1234, (c) $\mathrm{Hg}-1245$, and (d) La-112. In (b) and (d) dashed lines indicate $H_{c 2}^{a b}(T)$ from TDO measurements. In (d) we also illustrate $H_{c 2}^{c}$ for comparison. We note reasonable consistency among different experimental techniques, indicating strong suppression of $H^{*} \equiv H_{i r r}^{a b}(0)$ relative to $H_{c 2}^{a b}(0)$ (or $H_{p}$ ) in all cuprates. 

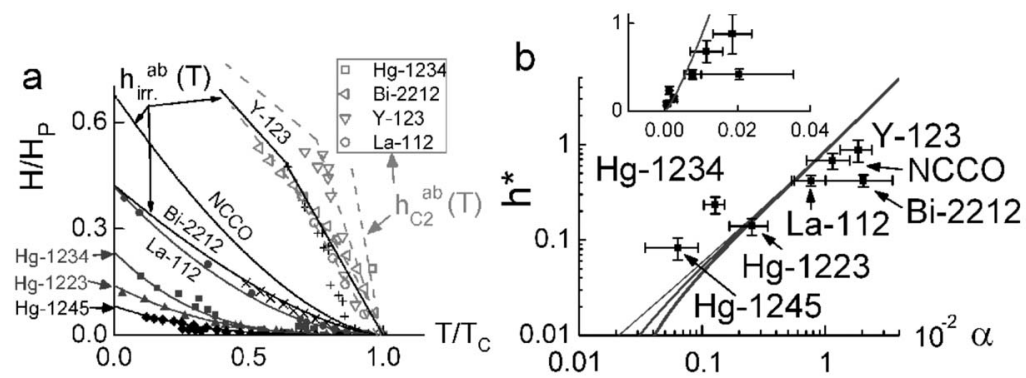

d

c

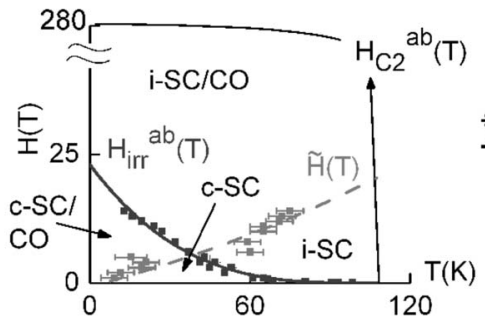

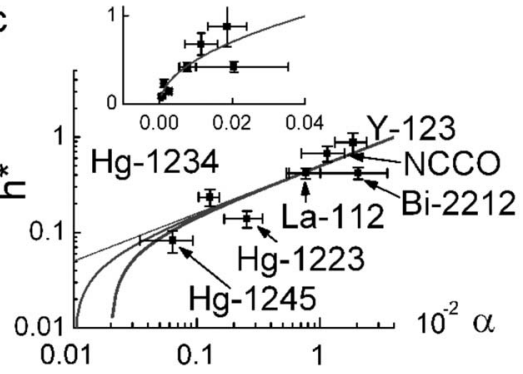

FIG. 3. (a) Reduced in-plane fields $\left(H_{i r r}^{a b} / H_{p}\right)$ and $\left(H_{c 2}^{a b} / H_{p}\right)$ vs $\left(T / T_{c}\right)$ for various cuprates. In the $T \rightarrow 0$ limit where $H_{i r r}^{a b} \rightarrow H^{*}$, the reduced fields $h^{*} \equiv\left(H^{*} / H_{p}\right)<1$ for all cuprates are listed in Table I for Y-123, NCCO, Bi-2212, La-112, $\mathrm{Hg}-1234$, Hg-1223, and Hg-1245 (in descending order). (b) $h^{*}$ vs $\alpha$ in logarithmic plot for different cuprates, with decreasing $\alpha$ representing increasing quantum fluctuations. The lines given by $-400\left|\alpha-\alpha_{c}\right| / \ln \left|\alpha-\alpha_{c}\right|$ represent the field-induced SDW scenario (Ref. 11) in Eq. (3) with different $\alpha_{c}=0,10^{-4}$, and $2 \times 10^{-4}$ from left to right. Inset: Linear plot of the main panel. (c) The same data as in (b) are compared with the power-law dependence (solid lines) given by $5\left(\alpha-\alpha_{c}\right)^{1 / 2}$, using different $\alpha_{c}=0,10^{-4}$, and $2 \times 10^{-4}$ from left to right. Inset: Linear plot of the main panel. (d) $H$ vs. $T$ diagram of $\mathrm{Hg}-1245$. (See text for details.) ity $H_{i r r}^{a b}$ among grains of varying orientation relative to the applied field. Examples of this consistency have been shown in Ref. 8 and also in the main panel and the inset of Fig. 1(a).

In addition to the four different cuprates considered in this work, we compare measurements of $H_{i r r}^{a b}(T)$ on other cuprate superconducting single crystals, including underdoped $\mathrm{YBa}_{2} \mathrm{Cu}_{3} \mathrm{O}_{7-\delta} \quad\left(\mathrm{Y}-123, T_{c}=87 \mathrm{~K}\right),{ }^{29}$ optimally doped $\mathrm{Nd}_{1.85} \mathrm{Ce}_{0.15} \mathrm{CuO}_{4} \quad\left(\mathrm{NCCO}, T_{c}=23 \mathrm{~K}\right),{ }^{30}$ and overdoped $\mathrm{Bi}_{2} \mathrm{Sr}_{2} \mathrm{CaCu}_{2} \mathrm{O}_{\mathrm{x}}\left(\mathrm{Bi}-2212, T_{c}=60 \mathrm{~K}\right){ }^{31}$ The irreversibility fields for these cuprates normalized to their corresponding paramagnetic fields $H_{p}$ are summarized in Fig. 3(a) as a function of the reduced temperature $\left(T / T_{c}\right)$, clearly demonstrating strong suppression of $H^{*}$ relative to $H_{p}$ and $H_{c 2}^{a b}(0)$ in all cuprates and implying significant field-induced quantum fluctuations.

The physical significance of $h^{*}$ may be better understood by considering how the magnetic irreversibility for $H \| a b$ occurs. For sufficiently low $T$ and small $H$, a supercurrent circulating both along and perpendicular to the $\mathrm{CuO}_{2}$ planes with a coherent superconducting phase can be induced and sustained, leading to magnetic irreversibility. On the other hand, strong thermal or quantum fluctuations due to large anisotropy and competing orders in the cuprates can reduce the phase coherence of supercurrents, particularly the coherence perpendicular to the $\mathrm{CuO}_{2}$ planes, thereby diminishing the magnetic irreversibility. Thus, we expect that the degree of in-plane magnetic irreversibility is dependent on the nominal doping level $\delta$, the electronic anisotropy $\gamma$, the number of $\mathrm{CuO}_{2}$ layers per unit cell, $n$, and the ratio of charge imbalance $^{23,24}\left(\delta_{o} / \delta_{i}\right)$ between the doping level of the outer layers $\left(\delta_{o}\right)$ and that of the inner layer(s) $\left(\delta_{i}\right)$ in multilayer cuprates with $n \geqslant 3$. In other words, $h^{*}$ for each cuprate superconductor may be expressed in terms of a material parameter $\alpha$ that depends on $\delta, \gamma, n$, and $\left(\delta_{o} / \delta_{i}\right)$, and the simplest assumption for a linearized dependence of $\alpha$ on these variables gives

$$
\alpha \equiv \begin{cases}\gamma^{-1} \delta\left(\delta_{o} / \delta_{i}\right)^{-(n-2)} & (n \geqslant 3) \\ \gamma^{-1} \delta & (n \leqslant 2)\end{cases}
$$

If the suppression of the in-plane magnetic irreversibility is associated with field-induced quantum fluctuations and the proximity to a quantum critical point $\alpha_{c},{ }^{11} h^{*}(\alpha)$ should be a function of $\left|\alpha-\alpha_{c}\right|$. Indeed, we find that using the empirically determined values for different cuprates tabulated in Table I and the definition of $\alpha$ given above, the $h^{*}$ vs. $\alpha$ data for a wide variety of cuprates appear to follow a trend, as shown in Fig. 3(b). For comparison, we include in Fig. 3(b) theoretical curves predicted for field-induced static spin density waves (SDWs) in cuprate superconductors in the limit of $H_{c 1}(0) \ll H \ll H_{c 2}(0)$, where $h^{*}$ (above which static SDWs coexist with $\mathrm{SC}$ ) satisfies the relation ${ }^{11}$

TABLE I. Quantum criticality parameters among different cuprates. All fields in tesla. $\sigma$ denotes a parameter's uncertainty.

\begin{tabular}{|c|c|c|c|c|c|c|c|c|c|c|c|c|c|}
\hline Compound & $\delta$ & $\delta_{o}$ & $\delta_{i}$ & $\gamma$ & $\sigma_{\gamma}$ & $\alpha\left(10^{-2}\right)$ & $\sigma_{\alpha}\left(10^{-3}\right)$ & $H^{*}$ & $\sigma_{H^{*}}$ & $H_{c 2}^{a b}\left[H_{P}\right]$ & $\sigma_{H_{P}}$ & $h^{*}$ & $\sigma_{h^{*}}$ \\
\hline Hg-1245 & 0.15 & 1.30 & 0.80 & 55 (Ref. 32) & 25 & 0.06 & 0.3 & 23.0 & 5.0 & {$[278]$} & 40 & 0.08 & 0.02 \\
\hline Hg-1223 & 0.15 & 1.04 & 0.92 & 52 (Ref. 33) & 18 & 0.26 & 0.9 & 48.5 & 6.5 & {$[347]$} & 50 & 0.14 & 0.02 \\
\hline Hg-1234 & 0.15 & 1.20 & 0.80 & 52 (Ref. 33) & 10 & 0.13 & 0.2 & 75.0 & 10.0 & {$[320]$} & 46 & 0.23 & 0.02 \\
\hline La-112 & 0.10 & 1.00 & 1.00 & 13 (Ref. 8) & 4.0 & 0.77 & 2.4 & 46.0 & 4.0 & $160[110]$ & 10 & 0.42 & 0.04 \\
\hline Bi-2212 & 0.225 & 1.00 & 1.00 & 11 (Ref. 31) & 8.0 & 2.05 & 15 & 65.0 & 10 & $100[155]$ & 22 & 0.42 & 0.06 \\
\hline $\mathrm{NCCO}$ & 0.15 & 1.00 & 1.00 & 13 (Ref. 30) & 5.0 & 1.15 & 4.4 & 40.0 & 5.0 & 77 [59] & 8.0 & 0.68 & 0.12 \\
\hline Y-123 & 0.13 & 1.00 & 1.00 & 7.0 (Ref. 33) & 2.0 & 1.86 & 5.3 & 210 & 50 & $600[239]$ & 25 & 0.88 & 0.23 \\
\hline
\end{tabular}




$$
h^{*}(\alpha) \propto\left|\alpha-\alpha_{c}\right| /\left(\ln \left|\alpha-\alpha_{c}\right|\right) .
$$

Here $\alpha_{c}$ is a nonuniversal critical point, ${ }^{11} h^{*}(\alpha) \rightarrow 0$ for $\alpha$ $\rightarrow \alpha_{c}$, and we have shown theoretical curves associated with three different $\alpha_{c}$ values for comparison with data. On the other hand, a simple scaling argument would assert a powerlaw dependence:

$$
h^{*}(\alpha) \propto\left|\alpha-\alpha_{c}\right|^{a} \quad(a>0) .
$$

Using $a \sim 0.5$ in Eq. (4), we compare the power-law dependence with experimental data in Fig. 3(c). This dependence appears to agree better with experimental data than the SDW-SC formalism in Eq. (3).

Although our available data cannot accurately determine $\alpha_{c}$, we further examine $\mathrm{Hg}-1245$ (which has the smallest $h^{*}$ ) for additional clues associated with the nature of the QCP. We find that the magnetization $M$ of $\mathrm{Hg}-1245$ always exhibits an anomalous increase for $T<\tilde{T}(H)$ [see the inset of Fig. 1(c)], indicating a field-induced reentry of magnetic ordering below $\widetilde{T}(H)$. This magnetism reentry line $\tilde{H}(T)$ is shown together with $H_{i r r}^{a b}(T)$ in Fig. 3(d). We suggest that the regime below both $H_{i r r}^{a b}$ and $\widetilde{H}$ corresponds to a coherent SC state (c-SC), and that bounded by $H_{i r r}^{a b}$ and $\widetilde{H}$ is associated with a coherent phase of coexisting $\mathrm{SC}$ and magnetic $\mathrm{CO}$ (c-SC-CO), whereas that above $H_{i r r}^{a b}$ is an incoherent SC phase ( $i$-SC and $i$-SC-CO) with strong fluctuations.

Our conjecture of a field-induced magnetic $\mathrm{CO}$ in $\mathrm{Hg}$ 1245 contributing to quantum fluctuations may be further corroborated by considering the $h^{*}$ vs. $\alpha$ dependence in the multilayered cuprates $\mathrm{Hg}-1223, \mathrm{Hg}-1234$, and $\mathrm{Hg}-1245$. While these cuprate superconductors have the highest $T_{c}$ and $H_{c 2}$ values, as shown in Table I, they also exhibit the smallest $h^{*}$ and $\alpha$ values, suggesting maximum quantum fluctuations. These strong quantum fluctuations can be attributed to both their extreme two-dimensionality ${ }^{27,34}$ (i.e., large $\gamma$ ) and significant charge imbalance that leads to strong $\mathrm{CO}$ in the inner layers. ${ }^{23,24}$ Indeed, muon spin resonance $(\mu \mathrm{SR})$ experiments ${ }^{35}$ have revealed increasing antiferromagnetic ordering in the inner layers of the multilayer cuprates with $n$ $\geqslant 3$. Given that the $\gamma$ values of all $\mathrm{Hg}$-based multilayer cuprates are comparable (Table I), the finding of larger quantum fluctuations (i.e., smaller $h^{*}$ ) in $\mathrm{Hg}$-1245 is suggestive of increasing quantum fluctuations with stronger competing order. However, further investigation of the $h^{*}$ and $\gamma$ values of other multilayer cuprates will be necessary to confirm whether competing orders in addition to large anisotropy contribute to quantum fluctuations.

In summary, our investigation of the in-plane magnetic irreversibility in a wide variety of cuprate superconductors reveals strong field-induced quantum fluctuations. The macroscopic irreversibility field exhibits dependences on such microscopic material parameters as the doping level, the charge imbalance in multilayered cuprates, and the electronic anisotropy. Our finding is consistent with the notion that cuprate superconductors are in close proximity to quantum criticality.

Research at Caltech was supported by NSF Grant No. DMR-0405088 and through the NHMFL. The SQUID data were taken at the Beckman Institute at Caltech. Work at Pohang University was supported by the Ministry of Science and Technology of Korea. The authors gratefully acknowledge Kazuyasu Tokiwa and Tsuneo Watanabe at the Tokyo University of Science for providing the $\mathrm{HgBa}_{2} \mathrm{Ca}_{4} \mathrm{Cu}_{5} \mathrm{O}_{\mathrm{x}}$ (Hg-1245) samples.
${ }^{1}$ D. S. Fisher, M. P. A. Fisher, and D. A. Huse, Phys. Rev. B 43, 130 (1991).

${ }^{2}$ G. Blatter et al., Rev. Mod. Phys. 66, 1125 (1994).

${ }^{3}$ N.-C. Yeh et al., Physica A 200, 374 (1993).

${ }^{4}$ L. Balents and D. R. Nelson, Phys. Rev. Lett. 73, 2618 (1994).

${ }^{5}$ T. Giamarchi and P. Le Doussal, Phys. Rev. B 52, 1242 (1995).

${ }^{6}$ G. Kotliar and C. M. Varma, Phys. Rev. Lett. 77, 2296 (1996).

${ }^{7}$ J. Kierfeld and V. M. Vinokur, Phys. Rev. B 69, 024501 (2004).

${ }^{8}$ V. S. Zapf et al., Phys. Rev. B 71, 134526 (2005).

${ }^{9}$ N.-C. Yeh et al., Int. J. Mod. Phys. B 19, 285 (2005).

${ }^{10}$ S.-C. Zhang, Science 275, 1089 (1997).

${ }^{11}$ E. Demler, S. Sachdev, and Y. Zhang, Phys. Rev. Lett. 87, 067202 (2001).

${ }^{12}$ S. Chakravarty, R. B. Laughlin, D. K. Morr, and C. Nayak, Phys. Rev. B 63, 094503 (2001).

${ }^{13}$ S. Sachdev, Rev. Mod. Phys. 75, 913 (2003).

${ }^{14}$ S. Kivelson et al., Rev. Mod. Phys. 75, 1201 (2003).

${ }^{15}$ P. A. Lee, N. Nagaosa, and X.-G. Wen, Rev. Mod. Phys. 78, 17 (2006).

${ }^{16}$ F. Onufrieva and P. Pfeuty, Phys. Rev. Lett. 92, 247003 (2004).

${ }^{17}$ C.-T. Chen, A. D. Beyer, and N.-C. Yeh, Solid State Commun. 143, 447 (2007).

${ }^{18}$ A. D. Beyer, C. T. Chen, and N.-C. Yeh, arXiv:cond-mat/0610855 (unpublished)
${ }^{19}$ C.-T. Chen and N.-C. Yeh, Phys. Rev. B 68, 220505(R) (2003).

${ }^{20}$ H. Y. Chen and C. S. Ting, Phys. Rev. B 71, 132505 (2005).

${ }^{21}$ B. Lake et al., Science 291, 1759 (2001).

${ }^{22}$ S. Chakravarty, H.-Y. Kee, and K. Volker, Nature (London) 428 , 53 (2004).

${ }^{23}$ H. Kotegawa et al., J. Phys. Chem. Solids 62, 171 (2001).

${ }^{24}$ H. Kotegawa et al., Phys. Rev. B 64, 064515 (2001).

${ }^{25}$ M. Naughton et al., Rev. Sci. Instrum. 68, 4061 (1997).

${ }^{26}$ D. S. Reed et al., Phys. Rev. B 51, 16448 (1995).

${ }^{27}$ M.-S. Kim et al., Phys. Rev. B 57, 6121 (1998).

${ }^{28}$ A. Iyo et al., Physica C 445, 17 (2006).

${ }^{29}$ J. L. O'Brien et al., Phys. Rev. B 61, 1584 (2000).

${ }^{30}$ N.-C. Yeh et al., Phys. Rev. B 45, 5710 (1992).

${ }^{31}$ L. Kruisin-Elbaum, T. Shibauchi, and C. H. Mielke, Phys. Rev. Lett. 92, 097005 (2004).

${ }^{32}$ The $\gamma$ value for $\mathrm{Hg}-1245$ was extrapolated from those of $\mathrm{Hg}$ 1201, Hg-1212, Hg-1223, and Hg-1234 (Ref. 33), with the latter three being comparable within experimental errors.

${ }^{33}$ D. Zech et al., Phys. Rev. B 53, R6026 (1996).

${ }^{34}$ M.-S. Kim, C. U. Jung, S. I. Lee, and A. Iyo, Phys. Rev. B 63, 134513 (2001).

${ }^{35}$ K. Tokiwa et al., Int. J. Mod. Phys. B 17, 3540 (2003). 